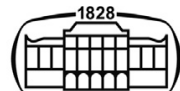

AKADÉMIAI KIADÓ

UNIVERSITY of DEBRECEN

\section{International Review of Applied Sciences and Engineering}

13 (2022) 1, 42-46

DOI:

$10.1556 / 1848.2021 .00288$

(c) 2021 The Author(s)

\title{
Modelling of density of states and energy level of chalcogenide quantum dots
}

\author{
M. Irshad Ahamed ${ }^{1 *} \odot$, Mansoor Ahamed ${ }^{2}$ and R. Muthaiyan ${ }^{3}$ \\ ${ }^{1}$ Department of Electronics and Communication, E.G.S. Pillay Engineering College, Nagapattinam- \\ 611002, Tamilnadu, India \\ 2 "Bimberg Chinese-German Centre for Green Photonics" of the Chinese Academy of Science at \\ Changchun Institute of Optics, Fine Mechanics and Physics, Changchun-130033, People's Republic of \\ China \\ ${ }^{3}$ Department of Electronics and Communication, University College of Engineering, Thirukkuvalai- \\ 610204, Tamilnadu, India
}

Received: April 15, 2021 • Accepted: June 27, 2021

Published online: August 20, 2021

ORIGINAL RESEARCH PAPER

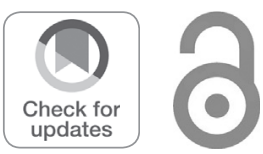

*Corresponding author.

E-mail: irshad_bcet@yahoo.co.in

\begin{abstract}
Quantum dots (QDs) or semiconductor nanocrystals are luminous materials with unique optical properties that can be fine-tuned by varying the size of the material. Chalcogenide QDs show strong quantum confinements effects owing to the fact that the exciton Bohr radius is much larger than the particle size, and tunable energy bandgap leads to widespread technological interest in near-infrared optical devices. In this communication, one dimensional $\mathrm{Cu}_{2} \mathrm{SnS}_{3}$ and $\mathrm{PbSe}_{\mathrm{x}} \mathrm{S}_{1-\mathrm{x}}$ QDs is modeled by a particle in a box model which was used to compute energies and density of states. The density of states and the energy level of QDs are determined as a function of the strengths of the potential walls of the inner box. The results exhibit that the density of states decreases exponentially with an increase in the energy level of QDs. The density of states at lower energy levels is more significant than what is observed in higher energy levels.
\end{abstract}

\section{KEYWORDS}

density of states, quantum dots, $\mathrm{Cu}_{2} \mathrm{SnS}_{3}, \mathrm{PbSe}_{\mathrm{x}} \mathrm{S}_{1-\mathrm{x}}$, energy level, chalcogenide

\section{INTRODUCTION}

Recent advances in light-emitting and detecting devices (LEDs and Photodetectors) layered structures fabrication technology have reached a level pushing the limits [1]; currently, in one or more of the three Cartesian directions, structures with nearly submicron sizes similar to the de Broglie wavelength of the electrons can be fabricated [2]. In telecommunication and optical computing applications, optoelectronic devices play a significant role and are extensively used. Optical switching schemes such as thermo-optic, electro-optic, and all other optic methods have been widely explored in optical communication networks, and on-chip interconnects [3].

In recent years, low-dimensional semiconductor materials have attracted extensive research interest in the field of optoelectronic device applications owing to their inherent structural properties such as ultra-small size and high active edge sites per unit mass [4]. Low-dimensional semiconductor materials have their own distinctive properties at the same time; they have typical applications such as optoelectronic devices. Based on the dimension, low-dimensional semiconductor materials can be classified as zero-dimensional (0D), onedimensional (1D), and two-dimensional (2D) depending on their nanoscale range in diverse spatial directions [5].

The two-dimensional nanomaterial is a layered structure. The conduction of electrons will be confined throughout the thickness of the material, but delocalization (electron free 
to move) is in a plane of sheet. The layers are bonded by Van der Waals forces and the atoms located in the in-plane are connected by covalent bonds [5, 6]. 2D materials attained an ultra-thin thickness at the atomic level, which sets them apart from conventional materials in dimension [7]. In one-dimensional materials, the electrons are confined to two dimensional spaces whereas delocalization occurs along the quantum rod/tube/wire axis. In the case of zero-dimensional nanomaterials, all three dimensions are fully confined, and electrons are not free to move. Hence, no delocalization occurs. Therefore, zero-dimensional nanomaterials electron movements are fully confined. Changes in morphology and spatial structure make zerodimensional nanomaterials exhibit versatility in chemical and physical properties [8].

The representatives in the family of low-dimensional semiconductor materials focus on nanodiamond, metal nanoparticles, quantum dots (QDs), graphene QDs are zerodimensional nanomaterials. Nano bars, nanoribbons, carbon nanotubes are one-dimensional nanomaterials, and graphene oxide, nanofilms, carbon nanowall are two-dimensional nanomaterials. These $1 \mathrm{D}$ and 2D nanomaterials offer unique advantages such as lightweight, extreme thinness, high transparency, high sensitivity, and the possibility for large-area fabrication. Along with obvious advantages, 1D and $2 \mathrm{D}$ nanomaterials have some disadvantages, which in some cases can significantly limit their application [9]. With such unique properties as tunable wavelength photoluminescence, high optical stability, chemical inertness, 0D nanomaterials offer great adaptability to optoelectronic applications such as light-emitting and detecting devices and solar cells. QDs with 3 dimensionally confined electrons constitute a crucial role of recent semiconductor studies [1012].

Luminous QDs are generally composed of I-IV-VI, IIVI, and I-III-V elements. The most important property of QDs is the large "surface to volume ratio," which results in discrete electronic states called "traps" on the surface. As a result of size confinement, the continuum of states in valence and conduction band is divided into discrete energy levels relating to energy bandgaps that are approximately and inversely proportional to the size of QDs radius $[13,14]$.

The density of states determines the various properties of nanomaterials, and this key freedom provides for designing and tuning nanomaterials for various applications. This phenomenon allows us to study the mechanical and optical properties of the same semiconductor nanomaterials with different sizes exhibiting various properties. For example, photoluminescence spectroscopy, thermal analysis, excitons in semiconductor nanocrystal, energy bandgap, etc., overall provide the ability to control the density of states and are significant for various applications of optoelectronic devices such as light-emitting devices, photodetector, biological devices, optical memories, and advanced photonic nanostructures. The density of states calculation is very helpful in investigating the electron transport properties of a system, since the variation in the density of states directly disturbs the electron transport properties of the system as a result of a reduction in size [15-18].

Recently Ilouno et al. $[19,20]$ reported about energy level and density of states variations of low dimensional quantum structures which are determined by using particle in a box model. Remarkably surface to volume ratio of the semiconductor nanostructures plays a significant role in investigating their properties. One of the foremost properties of semiconductor is density of states which plays a key role in the electronic properties of semiconductors. Golovatenko modeled the density of ground excitonic states of epitaxial growth binary alloy showing much wider emission and the photoluminescence peak is shifted towards higher energy level [21]. The modelling results suggested the effective energy transfer in the dense array of epitaxial growth QDs. Hence, it is important to study energy level and density of states variations of semiconductor nanostructures to better understand the opportunity.

Ternary $\mathrm{Cu}_{2} \mathrm{SnS}_{3} \& \mathrm{PbSe}_{\mathrm{x}} \mathrm{S}_{1-\mathrm{x}}$ chalcogenide QDs have been chosen in this research, for the application of light emitting and detecting devices and solar cells, due to their relatively low toxicity and availability in abundance. $\mathrm{Cu}_{2} \mathrm{SnS}_{3}$ is a promising substitute for quaternary systems, which provides an alternative to rare metal elements such as indium-based compounds and avoids a complex preparation process. $\mathrm{Cu}_{2} \mathrm{SnS}_{3}$ is a p-type semiconductor with high absorption coefficient value of $10^{4} \mathrm{~cm}^{-1}$ and with a bandgap range of $0.93-1.77 \mathrm{eV}$, making it a suitable absorber material for solar cell and lighting sensor applications. Similarly, ternary semiconductors such as PbSeS from the group of IVVI semiconductors are most commonly used in infrared optoelectronics. It offers potential uses for intermediate wavelength detectors and produces a unique response to cover wavelength ranging from 3,000 to 5,000 nm. Moreover, these materials show excellent optical absorption and emission properties. For solar cell applications, it exhibits excellent absorption and is highly compatible for designing light-emitting devices such as LDs, LEDs, and sensing devices [22-27].

\section{THEORY OF ANALYSIS}

The density of states are different energy states that electrons are allowed to occupy at a specific energy level, i.e., the number of electron states per unit energy per unit volume. The unit of density of states is expressed as $\mathrm{eV}^{-1} \mathrm{~cm}^{-3}$ and it offers information on how the energy states are distributed in a given solid. It is usually denoted as $g$ (E). Further, the density of states points to the availability of the number of states in a system which is important for determining the energy distribution of carrier and carrier concentration within a semiconductor [28].

Generally, in semiconductors, the motion of free electrons is limited with zero, one, and two spatial dimensions. Knowledge of the density of states of low dimensional nanostructures is required when applying semiconductor states to the systems of these dimensions. 
The number of states achieved by a quantum system is the possible number of available states, mathematically expressed as

$$
\varnothing(E)=\frac{V_{\text {system }}}{V_{\text {single state }}} \times N
$$

Where,

$\varnothing(E)$ represents the number of states, $V_{\text {system }}$ represents the volume of the whole systems (sphere, line, and circle), $V_{\text {single state }}$ represents the volume of a single state of the system, and $N$ is the number of atoms in the crystal.

In an excited state, the density of states depends on the energy gained by an electron. It is the first derivative of the state with respect to energy. It is mathematically expressed,

$$
g(E)=\frac{d \varnothing(E)}{d E}
$$

\subsection{Density of states of low dimensional systems}

One dimensional QDs are considered in the analysis of the energy level per unit energy per unit volume. It can be derived from the Schrodinger equation.

$$
\nabla^{2} \psi(x)+\frac{2 m^{*}(E-V)}{\hbar^{2}} \psi(x)=0
$$

Where, $m^{*}$ - Effective mass of the particle, $V$ - Volume of the system, $\psi(x)$ - Electron wave function, $\hbar$ - Reduced Planck's constant, $E$ - Energy, a real number

$$
\begin{gathered}
\nabla^{2}=\frac{\partial^{2}}{\partial x^{2}} \\
\nabla^{2} \psi(x)=\frac{\partial^{2} \psi(x)}{\partial x^{2}}
\end{gathered}
$$

Put Equation (5) in Equation (3)

$$
\frac{d^{2} \psi(x)}{d x^{2}}+\frac{2 m^{*}(E-V)}{\hbar^{2}} \psi(x)=0
$$

Put $\mathrm{V}=0$ in Equation (6)

$$
\begin{gathered}
\frac{d^{2} \psi(x)}{d x^{2}}+\frac{2 m E}{\hbar^{2}} \psi(x)=0 \\
\frac{2 m^{*} E}{\hbar^{2}}=K^{2}, \text { then } \mathrm{K}=\sqrt{\frac{2 m^{*} E}{\hbar^{2}}} \\
\frac{d^{2} \psi(x)}{d x^{2}}+K^{2} \psi(x)=0
\end{gathered}
$$

Where,

$$
\psi(x)=A \sin K x+B \cos k x
$$

Substituting $\mathrm{K}=\sqrt{\frac{2 m^{*} E}{\hbar^{2}}}$ in Equation (10)

$$
n=\frac{\sqrt{\frac{2 m^{*} E}{\hbar^{2}}} l}{\pi}=\left(2 m^{*} E\right)^{\frac{1}{2}} \frac{l}{\hbar \pi}
$$

The density per unit energy is obtained by using chain rule as given below,

$$
\frac{d N}{d E}=\frac{d N}{d K} \frac{d k}{d E}=\frac{\left(2 m^{*} E\right)^{-1 / 2} \times m l}{\hbar \pi}
$$

The density of states per unit energy per unit volume divided by the volume of the crystal,

$$
\begin{gathered}
g(E)=\frac{\frac{\left(2 m^{*} E\right)^{-1 / 2} \times m^{*} l}{\hbar \pi}}{l}=\frac{\left(2 m^{*} E\right)^{-1 / 2} \times m^{*}}{\hbar \pi}=\frac{m^{*}}{\sqrt{2 m E} \pi \hbar} \\
g(E)=\frac{1}{\pi \hbar} \sqrt{\frac{m^{*}}{2 E}}
\end{gathered}
$$

The above equation shows that the density of states for QDs depends on energy [17].

\section{RESULTS AND DISCUSSION}

Quantum confinement effects constitute the unique property of QDs, which becomes discrete the density of states near the band edges with the ability to determine the spacing between the energy bands in semiconductors. A simple theoretical model has been formulated to study the variation of density of states with respect to increase in the energy level of QDs.

Figures 1 and 2 show the density of states as a function of the energy of $\mathrm{Cu}_{2} \mathrm{SnS}_{3}$ and $\mathrm{PbSe}_{\mathrm{x}} \mathrm{S}_{1-\mathrm{x}}$ QDs. The result reciprocates an exponential decrease in the density of states with an increase in energy levels of QDs. The density of states of lower energy levels is more significant than what is observed at higher energy levels. This energy variation in $\mathrm{PbSe}_{\mathrm{x}} \mathrm{S}_{1-\mathrm{x}}$ QDs is much higher than $\mathrm{Cu}_{2} \mathrm{SnS}_{3}$ QDs, which is due to the effective mass of electrons in the compound.

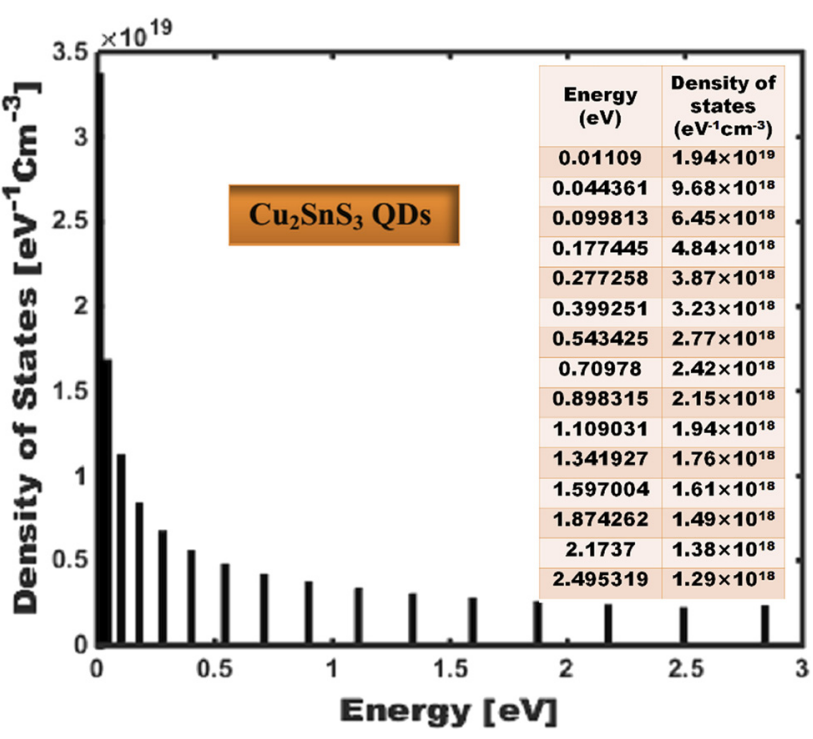

Fig. 1. Variation of the density of states with respect to the energy level of $\mathrm{Cu}_{2} \mathrm{SnS}_{3}$ QDs 


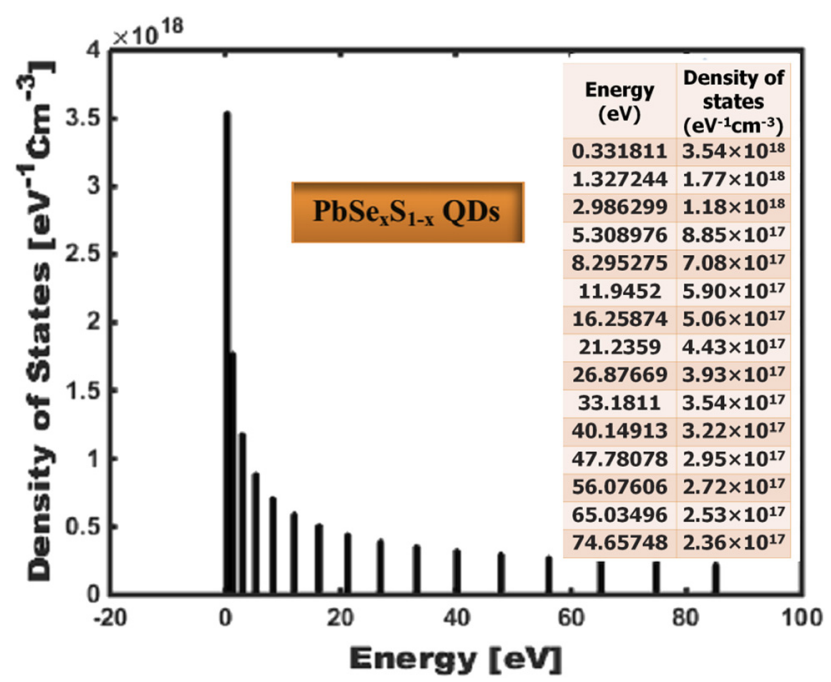

Fig. 2. Variation of the density of states with respect to the energy level of $\mathrm{PbSe}_{\mathrm{x}} \mathrm{S}_{1-\mathrm{x}}$ QDs

The density of states and the energy level spacing change with a reduction in material size as well as a constituent element in the ternary alloy and temperature. It also indicates the dependence of the density of states on the quantum number with respect to energy. The most important characteristic of the low dimensional nanostructure is their density of states which shows a sharp dependence on energy level, especially QDs systems.

The density of states is remarkably kindred to the optoelectronic properties of a semiconductor nanostructure through its due absorption coefficient, which directly affects the characteristics of the optoelectronic devices. Hence these parameters significantly affect the energy band.

In the case of $\mathrm{PbSe}_{\mathrm{x}} \mathrm{S}_{1-\mathrm{x}} \mathrm{QDs}$, the effective mass of the electron is much lower $\left(9.276 \times 10^{-32} \mathrm{~kg}\right)$, almost equivalent to the mass of an electron at rest. In return, the corresponding energy level variation is high. At the same time, in $\mathrm{Cu}_{2} \mathrm{SnS}_{3} \mathrm{QDs}$, the effective mass electron is in the range between $2.18 \times 10^{-30} \mathrm{~kg}$ and $2.78 \times 10^{-30} \mathrm{~kg}$, and the energy level is relatively low, resulting in a high density of states. Energy bandgap is not much affected by $\mathrm{Cu}_{2} \mathrm{SnS}_{3}$ QDs at lower energy levels, which confirms the stability of the material.

\section{CONCLUSION}

$\mathrm{Cu}_{2} \mathrm{SnS}_{3}$ and $\mathrm{PbSe}_{\mathrm{x}} \mathrm{S}_{1-\mathrm{x}}$ QDs have been successfully modeled with respect to energy level and density of states by the particle in a box model. It is reported from the theory that energy level increases with a decrease in the density of states. Furthermore, it has been found that the low effective mass of electrons and holes in semiconductor nanomaterials may not vary the density of states at a higher level. This model would highly contribute to calculating and understand the electronic properties of various nanomaterials.

\section{ACKNOWLEDGEMENT}

The authors wish to thank Dr. E. Edward Anand, Professor, E.G.S. Pillay Engineering college for his technical guidance and support during this work.

\section{REFERENCES}

[1] Q. Ma, G. Ren, A. Mitchell, and J. Ou, "Recent advances on hybrid integration of 2D materials on integrated optics platforms," Nanophoton, vol. 9, pp. 2191-214, 2020.

[2] D. Li, K. Jiang, X. Sun, and C. Guo, "AlGaN photonics: recent advances in materials and ultraviolet devices," Adv. Opti. Photon., vol. 10, p. 43, 2018.

[3] K. Xu, B. Y. Zhang, Y. Hu, M.W. Khan, R. Ou, Q. Ma, C. Shangguan, et al. "A high-performance visible-light-driven all-optical switch enabled by ultra-thin gallium sulfide," J. Mater. Chem. C, Mater. Opt. Electron. Devices, vol. 9, no. 9, pp. 3115-21, 2021.

[4] Z. Wang, T. Hu, R. Liang, and M. Wei, "Application of zerodimensional nanomaterials in biosensing," Front. Chem., vol. 8, 2020. https://doi.org/10.3389/fchem.2020.00320.

[5] J. Fang, Z. Zhou, M. Xiao, Z. Lou, Z. Wei, and G. Shen, "Recent advances in low-dimensional semiconductor nanomaterials and their applications in high-performance photodetectors," Info Mat, vol. 2, no. 2, pp. 291-317, 2019. https://doi.org/10.1002/inf2. 12067.

[6] Q. Ma, G. Ren, K. Xu, and J. Ou, "Tunable optical properties of 2D materials and their applications," Adv. Opt. Mater., vol. 9, no. 2, p. 2001313, 2020. https://doi.org/10.1002/adom.202001313.

[7] K. Khan, et al., "Recent developments in emerging two-dimensional materials and their applications," J. Mater. Chem. C, vol. 8, no. 2, pp. 387-440, 2020. https://doi.org/10.1039/c9tc04187g.

[8] F. A. Michael, J. F. Paulo, and L. S. Daniel, Eds. Nanomaterials and Nanotechnologies and Design: An Introduction for Engineers and Architect, Armsterdam: Elsevier, 2009. Available from: Elsevier books.

[9] G. Korotcenkov, "Current trends in nanomaterials for metal oxide-based conductometric gas sensors: advantages and limitations. Part 1: 1D and 2D nanostructures," Nanomaterials, vol. 10, no. 7, p. 1392, 2020. https://doi.org/10.3390/nano10071392.

[10] F. Rodríguez-Mas, J. Ferrer, J. Alonso, D. Valiente, and S. Fernández de Ávila, "A comparative study of theoretical methods to estimate semiconductor nanoparticles' size," Crystals, vol. 10, p. 226, 2020.

[11] M.I. Ahamed, and K.S. Kumar, "Modelling of electronic and optical properties of $\mathrm{Cu} 2 \mathrm{SnS} 3$ quantum dots for optoelectronics applications," Mat.Sci.-Poland, vol. 37, pp. 108-15, 2019.

[12] S. Dias, K. Kumawat, S. Biswas, and S. Krupanidhi, "Heat-up synthesis of $\mathrm{Cu} 2 \mathrm{SnS} 3$ quantum dots for near infrared photodetection," RSC Adv., vol. 7, pp. 23301-8, 2017.

[13] S. Nath, D. Chakdar, G. Gope, and D. Avasthi, "Effect of $100 \mathrm{MeV}$ nickel ions on silica coated ZnS quantum dots," J. Nanoelectro.Optoelectro., vol. 3, pp. 180-3, 2008.

[14] D. Mohanta, S. Nath, A. Bordoloi, A. Choudhury, S. Dolui, and N. Mishra, "Optical absorption study of $100-\mathrm{MeV}$ chlorine ion-irradiated hydroxyl-free $\mathrm{ZnO}$ semiconductor quantum dots," J. Appl. Phy., vol. 92, pp. 7149-52, 2002. 
[15] M. Omar, Introduction to Nanomaterials and Devices. [e-book]. New Jersey, Wiley, 2011, Available through: Wiley Online Library https://onlinelibrary.wiley.com/doi/book/10.1002/9781118148419.

[16] J. Heremans, "Low-dimensional thermoelectricity," Acta Physica Pol. A, vol. 108, pp. 609-34, 2005.

[17] S. Lee, N. Shin, J. Ko, M. Park, and R. Kummel, "Density of states of quantum dots and crossover from 3D to Q0D electron gas," Semiconductor Sci. Technol., vol. 7, pp. 1072-9, 1992.

[18] K. Langfeld, B. Lucini, A. Rago, R. Pellegrini, and L. Bongiovanni, "The density of states approach for the simulation of finite density quantum field theories," J. Phys. Conf. Ser., vol. 631, p. 012063, 2015.

[19] J. Ilouno, I. Audu, M. Mafuyai, and N. Okpara, Asian J. Res. Rev. Phys., vol. 1, pp. 1-6, 2018.

[20] J. Ilouno, N. Okapara, and M. Mafuyai, Int. J. Sci. Eng. Tech. Res., vol. 7, pp. 364-75, 2018.

[21] A. Golovatenko, M. Semina, A. Rodina, and T. Shubina, "Density of states and photoluminescence spectra in the dense arrays of epitaxial CdSe/ZnSe quantum dots with Gaussian potential profile," Acta Physica Pol. A, vol. 129, pp. A-107-A-110, 2016.

[22] S. Dias, K. Kumawat, S. Biswas, and S. Krupanidhi, "Solvothermal synthesis of $\mathrm{Cu} 2 \mathrm{SnS} 3$ quantum dots and their application in near- infrared photodetectors," Inorg. Chem., vol. 56, pp. 2198-203, 2017.

[23] K. M. H. Shamima, G. Ram, and V. Kamlanathan, "Influence of solvents on solvothermal synthesis of Cu2SnS3 nanoparticles with enhanced optical, photoconductive and electrical properties,"Mat. Technol., vol. 33, pp. 72-8, 2017.

[24] M. Irshad Ahamed, and K. Sathish Kumar, "Studies on Cu2SnS3 quantum dots for O-band wavelength detection," Mat. Sci.Poland, vol. 37, pp. 225-9, 2019.

[25] S. Dias, and S. Krupanidhi, "Solution processed Cu2SnS3 thin films for visible and infrared photodetector applications," AIP Adv., vol. 6, p. 025217, 2016.

[26] S. Jathar, et al. "Ternary Cu2SnS3: synthesis, structure, photoelectrochemical activity, and heterojunction band offset and alignment," Chem. Mater., vol. 33, no. 6, pp. 1983-93, 2021. https://doi.org/10.1021/acs.chemmater.0c03223.

[27] M.I. Ahamed, K.S. Kumar, E.E. Anand, and A. Sivaranjani, “Optical attenuation modelling of $\mathrm{PbSexS}_{1-\mathrm{x}}$ quantum dots for optoelectronic applications," J. Ovonic Res., vol. 16, pp. 245-52, 2020.

[28] Density of states, gerogia institute of technology. Available: http:// alan.ece.gatech.edu/ECE6451/Lectures/StudentLectures/King_ Notes_Density_of_States_2D1D0D.pdf. [Accessed: Jan. 24, 2021]. 